\title{
Facial Reactions to Emotional Facial Expressions: Affect or Cognition?
}

\author{
Ursula Hess \\ University of Quebec at Montreal, Canada \\ Pierre Philippot \\ University of Louvain-La-Neuve, Louvain, Belgium \\ Sylvie Blairy \\ University of Quebec at Montreal, Canada
}

\begin{abstract}
This study investigated whether observers' facial reactions to the emotional facial expressions of others represent an affective or a cognitive response to these emotional expressions. Three hypotheses were contrasted: (1) facial reactions to emotional facial expressions are due to mimicry as part of an affective empathic reaction; (2) facial reactions to emotional facial expressions are a reflection of shared affect due to emotion induction; and (3) facial reactions to emotional facial expressions are determined by cognitive load depending on task difficulty. Two experiments were conducted varying type of task, presentation of stimuli, and task difficulty. The results show that depending on the nature of the rating task, facial reactions to facial expressions may be either affective or cognitive. Specifically, evidence for facial mimicry was only found when individuals made judgements regarding the valence of an emotional facial expression. Other types of judgements regarding facial expressions did not seem to elicit mimicry but may lead to facial responses related to cognitive load.
\end{abstract}

\section{INTRODUCTION}

The expression of emotions plays an important part in human interaction. Emotion displays may either express a sender's emotional state (Cacioppo, Petty, Losch, \& Kim, 1986; Fridlund, Ekman, \& Oster, 1987; Hess, Kappas,

Requests for reprints should be sent to Dr Ursula Hess, Department of Psychology, University of Quebec at Montreal, P.O. Box 8888, Station A, Montreal, QC H3C 3P8, Canada.

This research was funded by grant from the Fonds pour la formation des chercheurs et l'aide à la recherche (FCAR) and the University of Quebec at Montreal (PAFACC) to the first author and a grant from the Fond National de la Recherche Scientifique to the second author. We thank Patrick Bourgeois, Joelle Couture, Pedro Herrera, Erik Coats, Emmanuelle Zech, Sibylle Dozier, and Janique Sangsue for their help in collecting the data and François Labelle for his help in preparing the stimuli. 
\& Banse, 1995) or signal a listener's understanding of the sender's feelings (e.g. Bavelas, Black, Lemery, \& Mullett, 1986; Krause, 1990). In fact, in cases where the nonverbal exchange of emotional information encounters difficulties (as is the case for some schizophrenics), a breakdown of interaction patterns for even the most trivial of interactions may result (Krause, 1990).

Congruent with the significance of this domain, a large body of research has been accumulated regarding emotional displays (see e.g. Feldman \& Rimé, 1991). Whereas early research focused mainly on whether emotional facial expressions can be reliably decoded (Ekman, Friesen, \& Ellsworth, 1972), research has recently moved towards the investigation of nonverbal emotional exchange from a more interactional perspective (e.g. Patterson, 1991). Within this general trend, issues such as emotion contagion (Hatfield, Cacioppo, \& Rapson, 1992), empathy (Eisenberg \& Fabes, 1990), and counter-transference (Hsee, Hatfield, \& Chemtob, 1992; Krause \& Lütolf, 1988) have received the attention of empirical researchers.

In this general context, studies focusing on the observer, and more specifically on the observer's facial reactions to the emotional facial expressions of the sender, have gained prominence (e.g. Bush, Barr, McHugo, \& Lanzetta, 1989; Dimberg, 1982; Wallbott, 1991). These studies have documented that observers tend to show emotional facial expressions that are congruent with the expressions shown by the sender. This finding has some important implications for a related field: Empathy defined as shared effect. For example, Eisenberg and Strayer (1987) define empathy as an affective state that stems from the apprehension of another's emotional state or condition and that is congruent with it. In this context, congruent facial responses, that is, facial motor mimicry, have been considered an integral part of the empathic response (e.g. Basch, 1983; Bavelas, Black, Lemery, \& Mullett, 1987; Hoffman, 1984).

The goal of the present article is to investigate the claim that congruent emotional facial expressions are part of an affective response to the emotional expressions of the sender. For this, three hypotheses proposing different underlying processes to account for mimicry of the emotional expressions of others will be tested. Below, the rival hypotheses will be described in more detail.

The role of nonverbal responses to the emotion displays of others in the context of shared affect betwen sender and observer was first addressed by Lipps (1907). Specifically, he proposed that the observation of emotion displays leads to mimicry, which in turn elicits a congruent emotional state in the observer. Lipps proposed a model according to which individuals tend to imitate the emotional displays of their interaction partners. These imitated behaviours elicit_via a feedback process-corresponding emo- 
tional states. The observers relate their own feeling state to their knowledge about emotional experiences and attribute the emotional state to the interaction partner. In summary, imitation leads to shared affect which facilitates emotion recognition (Wallbott, 1991).

In recent years, Lipps' notions have seen a renaissance and a similar notion has recently been expressed in the general context of research on empathy and contagion. Hatfield et al. (1992, pp. 153-154) define "primitive contagion" as "the tendency to automatically mimic and synchronize expressions, vocalizations, postures, and movements with those of another person and, consequently, to converge emotionally". In this conceptualisation, it is assumed that afferent feedback from the imitated movements will induce a corresponding emotion which might be used by the observer to infer the sender's emotional state (Hsee et al., 1992). Laird et al. (1994), who also propose a process whereby mimicry leads to emotional contagion, suggest a causal process congruent with self-perception theory (Bem, 1972) or with James' (1890) peripheral theory.

Empirical evidence for Lipps' notions, as well as related, more recent proposals, stems from a number of different sources. First, several studies have confirmed that exposure to emotional facial expression elicits selfreports of congruent affect in observers (e.g. Hsee et al., 1992; Laird et al., 1994; Schneider, Gur, Gur, \& Muenz, 1994; Strayer, 1993).

Second, evidence for facial mimicry has been reported both for infants (e.g. Meltzoff, 1990) and for adults (e.g. Dimberg, 1982; Kappas, Hess, \& Banse, 1992; Wallbott, 1991). In addition, there is evidence that individuals tend to imitate other aspects of nonverbal emotion displays such as nonemotional movement (e.g. tensing of arm muscles when watching arm wrestling; Berger \& Hadley, 1975) or an interaction partner's speech style and posture, especially when they like the interaction partner (see e.g. Bernieri \& Rosenthal, 1991).

Third, the suggested causal link between facial expression and experience emotion-afferent feedback from the face-has been supported. Recent reviews (Manstead, 1988; Matsumoto, 1987; Winton, 1986) agree in so far as a dimensional view of the monotonicity version of the Facial Feedback Hypothesis is concerned; specifically, self- or experimenter-induced increases or decreases in emotional facial expressions tend to increase or decrease the experienced emotional state. In addition, just as there is evidence for the imitation of a variety of nonverbal emotion displays, there is evidence that the induction of patterned expressive postural displays (Duclos et al., 1989; Stepper \& Strack, 1993), vocal displays (Hatfield et al., 1995), and respiration rhythms (Bourgeois et al., 1995; Philippot, Chapelle, \& Blairy, 1994) influence the experienced affective state in a manner similar to facial feedback. 
Fourth, it has been shown that the ability to decode another person's emotional state is related to physiological linkage ${ }^{1}-$ which might be interpreted in terms of shared affect (Levenson \& Ruef, 1992).

Most of the studies reviewed above have focused on facial emotional displays which have been found to be especially salient sources of emotional information (Hess, Kappas, \& Scherer, 1988; Noller, 1985). Thus, although we regard mimicry as not being limited to the face, the following considerations will focus on facial displays and their effect on the observer.

In sum, a series of studies offer evidence suggesting that: (a) the exposure to another person's facial emotional displays induces mimicry; (b) self-induced emotional displays elicit-via a feedback process-a congruent emotional state in the observer; and (c) shared affect, or at least its physiological aspect, may be linked to increased decoding accuracy.

It is important to note that no study has evaluated the complete chain of mediation proposed. And, it is indeed difficult to conceive of a study that could test, without serious demand problems, the ensemble of causal links proposed. However, while the indirect chain of evidence presented earlier supports the contention that mimicry is related to emotion recognition, a number of problems have to be addressed.

First, in most cases where evidence for induction of congruent affect was found, series of the same expressions were shown to the observer (e.g. Hsee et al., 1992; Laird et al., 1994; Schneider et al., 1994). This leads to the alternative hypothesis that exposure to the emotional facial expressions of the target persons worked as an emotion induction procedure similar to the Velten technique (Velten, 1968), which is designed to induce happy and sad moods. That is, the repetitive exposure to facial stimuli of a similar emotional nature may have an effect equivalent to the effect of the repetitive exposure to phrases of a similar emotional nature. In this case, contagion would be independent of mimicry. Indeed, none of the studies quoted earlier has shown that the emotional feeling state induced by exposure to facial expressions was mediated by facial mimicry and facial feedback.

However, when looking at anger expressions in particular, a second alternative hypothesis can be advanced, which suggests that congruent facial expression to a sender's emotion display are not part of an affective but rather of a cognitive response. Specifically, it is possible that neither physiological linkage nor facial mimicry is part of a shared effect. That is, the observed synchronisation may be due to time-locked but independent events, which happen at the same point in time but are psychologically

Physiological linkage refers to the correlation of the sender's physiological reactions with the decoder's physiological reactions. Levenson and Ruef (1992) found higher correlations between sender and decoder to be related to better decoding accuracy. 
different. For example, while the target person shows an expression of anger, the decoder frowns in response to the greater task difficulty of decoding anger expressions (Darwin, 1862; Smith, 1989). Thus, the facial expressions shown by the observer are congruent with the target's expressions but are caused by a psychologically different process linked to the processing of the stimuli during the decoding task.

Hence, three different processes could account for facial responses to facial expressions: (i) primary mimicry as defined by Lipps (1907) or Hatfield et al. (1992) (Mimicry Hypothesis); (ii) direct emotion induction by a Velten-type process (Mood Induction Hypothesis); and (iii) responses to task characteristics such as decoding difficulty (Difficulty Hypothesis). While the first two hypotheses interpret facial reactions to emotion displays as a marker of an affective reaction, the third hypothesis views them as a marker of a cognitive process. However, these different processes are not mutually exclusive. It is both possible and likely that observers mimic the emotional facial expressions of others while at the same time showing facial reactions to the characteristics of the task or experiencing an affective state induced by repeated exposure to an emotional expression.

In summary, observers' facial reactions to a target's facial expressions may have important implications for their interaction. Further, a number of different processes may be implicated and may have differing influences in a given situation. Consequently, the disentanglement of the processes governing facial reactions to facial expressions is of considerable theoretical importance. This is the goal of the present paper.

\section{OVERVIEW}

Two studies were conducted to test the three hypotheses outlined above by manipulating: (a) the emotional valence of the stimuli (anger vs. happiness expressions); (b) the stimulus presentation (presentation within a block in which all stimuli were of the same emotional nature vs. presentation in random order); (c) the intensity of the expressions; and (d) task difficulty.

Experiment 1 addressed the question of whether congruent facial reactions to facial expressions are due to emotion induction via the repeated presentation of the same type of emotional expression or rather to another process linked to emotion recognition. For this, a standard emotion recognition task was employed; participants were asked to rate a series of emotional facial expressions regarding the emotional state expressed by the sender. According to the Mood Induction Hypothesis, emotion induction should be more likely to occur and to lead to congruent facial expressions when the participant is exposed to a series of expressions of 
the same emotion. Yet, other processes linked to the recognition task should not be influenced by the order of presentation. Thus, expressions were presented either in a random order or as series of eight expressions of the same emotion.

Further, we varied the intensity of the emotional facial expressions. Previous studies that found evidence for facial mimicry employed strong prototypical expressions which are very easy to decode (e.g. Dimberg, 1987; Wallbott, 1991). If congruent facial expressions are due to a reflex-like mirroring of the observed expression (Mimicry Hypothesis), more intense mimicry should be observed for intense expressions than for weak ones.

Finally, if the Difficulty Hypothesis is correct, Corrugator Supercilii muscle activity (as an index of frowning) due to task difficulty should be more likely in response to weaker expressions, as well as to expressions of anger, because they are difficult to decode. Further, Zygomaticus Major muscle activity (as an index of smiling) should be more likely in response to stronger expressions and to expressions of happiness, because they are easy to decode.

Experiment 2 was conducted to assess the impact of task difficulty, both for series of expressions and for mixed presentations. Given the confound between stimulus valence and task difficulty (positive facial expressions are easier to decode than negative facial expressions), it was necessary to manipulate these variable independently to test the task difficulty hypothesis adequately. Table 1 summarises the expected findings according to the three hypotheses just outlined.

TABLE 1

Predictions According to the Three Competing Hypotheses

\begin{tabular}{|c|c|c|c|}
\hline \multirow[b]{2}{*}{ Conditions } & \multicolumn{3}{|c|}{ Hypotheses } \\
\hline & Mood Induction & Difficulty & Mimicry \\
\hline $\begin{array}{l}\text { Block of } \\
\text { expressions vs. } \\
\text { random order }\end{array}$ & $\begin{array}{l}\text { More congruent } \\
\text { expressions for block } \\
\text { than for random } \\
\text { presentation }\end{array}$ & No difference & No difference \\
\hline $\begin{array}{l}\text { Difficult to decode } \\
\text { expressions }\end{array}$ & $\begin{array}{l}\text { More congruent } \\
\text { expressions for block } \\
\text { presentation }\end{array}$ & $\begin{array}{l}\text { Frown }=>\text { more } \\
\text { Corrugator } \\
\text { Supercilii activity }\end{array}$ & $\begin{array}{l}\text { Emotion-congruent } \\
\text { expressions }\end{array}$ \\
\hline $\begin{array}{l}\text { Easy to decode } \\
\text { expressions }\end{array}$ & $\begin{array}{l}\text { More congruent } \\
\text { expressions for block } \\
\text { presentation }\end{array}$ & $\begin{array}{l}\text { Relaxation of } \\
\text { Corrugator } \\
\text { Supercilii }\end{array}$ & $\begin{array}{l}\text { Emotion-congruent } \\
\text { expressions }\end{array}$ \\
\hline
\end{tabular}




\section{EXPERIMENT 1}

\section{Method}

\section{Participants}

Forty-eight female volunteers, 22 in the mixed condition and 26 in the block condition, were recruited at the University of Quebec at Montreal.

\section{Stimuli}

Expressions of anger, happiness and disgust were employed. The disgust expression served as filler items and data recorded during presentation of these expressions were not analysed. Four full-blown emotion displays of happiness, anger, and disgust, portrayed by two female and two male Caucasian actors were selected from a series of standardised emotional facial expressions (JACFEE; Matsumoto \& Ekman, 1988). The expressions were digitised and translated from colour to black and white. Based on these emotional facial expressions and the neutral faces of the same actors, we created a set of weak emotional facial expressions and a set of strong emotional facial expressions. This was achieved by interpolating between the neutral and the emotional expressions, using the program Morph 1.0. For this, corresponding points on the face outline, eye brows, eyes, nose, lips, etc., were marked on the neutral and the emotional facial expressions. Morph then interpolates between the corresponding points on the two original images. The resulting 24 emotional facial expressions ( 3 emotions $\times 4$ actors $\times 2$ intensities) were presented using an Apple Macintosh Centris 610 .

For the block condition the expressions were presented as three series of eight expressions each. The order of the series was counterbalanced over participants. Within each series the four weak emotional facial expressions were always presented before the four strong expressions. For the mixed condition, the 24 facial expressions were presented either in a random order or its reverse

\section{Dependent Measures}

Facial EMG. Facial EMG was measured on the left side of the face. Activity of the Zygomaticus Major (cheek) was employed to assess smiling and activity of the Corrugator Supercilii (eyebrow) was employed to assess frowning. Muscular activity was measured using bipolar placements of Med Associates Inc. $\mathrm{Ag} / \mathrm{AgCl}$ miniature surface electrodes with Med Associates Inc. electrolyte gel (TD41). The skin was 
cleansed with PDI disposable electrode preparation pads $(70 \%$ alcohol and pumice). Electrode placements were chosen according to Fridlund and Cacioppo (1986). A Contact Precision Instruments system with $60 \mathrm{~Hz}$ notch filter was used to amplify the raw EMG signals, which were integrated with $200 \mathrm{msec}$ time constant. The smoothed EMG signal was sampled at $10 \mathrm{~Hz}$ and stored to disk. ${ }^{2}$

Ratings. Following the presentation of each stimulus, participants were asked to assess the emotions portrayed as well as their intensities. For this, participants indicated the degree to which the expression reflected each of the following seven emotions: happiness, sadness, fear, anger, disgust, surprise, contempt. Using the mouse, participants clicked a point on each emotion scale indicating the intensity with which the face reflected that specific emotion. The scales were represented by a 200 pixels long, bounded rectangle on the screen and the pixels were graded in colour from light gray to dark gray, with the darker end of the scale indicating greater intensity of the emotion. Each scale contained an emotion label and was anchored with the verbal labels "not at all" and "very intensely". In addition, participants indicated how difficult the task was on a scale labelled "task difficulty", which had the same format as the emotion scales.

\section{Procedure}

The experimenter explained to the participants that their task would be to judge the emotion(s) portrayed by a series of stimulus persons. They were informed that during the experiment physiological measures would be taken. To reduce the possibility that participants were aware that we were interested in their facial expressions we employed a cover story suggesting that the experiment was concerned with frontal brain activity and that the transducers affixed were EEG electrodes. Furthermore, participants were informed that they would be filmed during the experiment. To avoid participants focusing on the camera during the experiment, the video camera was hidden. Participants who signed a consent form were seated in a comfortable armchair and the electrodes were attached. In order to familiarise the participants with the rating task they were asked to complete two practice trials during which the experimenter answered questions regarding the procedure.

\footnotetext{
In addition, skin conductance level and inter-beat intervals were measured. This also applied to Experiments $2 \mathrm{a}$ and $2 \mathrm{~b}$ (see later). The results pertaining to these measures will not be reported in the framework of this article.
} 
On each trial the participants first saw the neutral face of the stimulus person followed by the emotional facial expression of the same person. Following this, the rating scales were presented. Each face was shown for 10 seconds with a 5 -second interval between faces. Baselines were recorded during the 5 seconds before each trial. At the end of the experiment the participants were interviewed regarding their hypotheses. They were then fully debriefed and any remaining questions were answered by the experimenter. Finally, they were asked for their consent for use of the video records.

\section{Artefact Control and Data Reduction}

The video records for all participants were inspected for movements that could disrupt the psychophysiological measures. Using a visual editing computer program Physio3 (Banse, 1995), periods corresponding to such movements were set missing and excluded from further analyses.

It is possible that observers may show facial reactions to the stimulus persons (as a function of physical attractiveness, etc.) Because the current analyses focus only on facial reactions to emotional facial expressions it is important to not confound reactions to the stimulus person with reactions to the emotional facial expressions. Consequently, for the purpose of the following analyses, the periods during which the participants saw the neutral face of the stimulus person served as baselines for the EMG measures. Standardised difference scores were calculated for each trial. All analyses reported were based on these means.

\section{Results}

\section{Analyses}

The dependent variables were entered into a 2 (Emotion: angry vs. happy; within-subjects) $\times 2$ (Intensity of expression: strong vs. weak; within-subjects) $\times 2$ (mixed vs. block Presentation; between-subjects) mixed model analysis of variance using a multivariate approach.

\section{Task Difficulty}

Main effects of Emotion and Intensity, $[F(1,43)=19.24, P<.001]$ and $F(1,43)=35.53, P<.001]$, respectively, as well as an Emotion $\times$ Intensity and a Presentation $\times$ Emotion $\times$ Intensity interaction $[F(1,43)$ $=4.40, P=.042$ and $F(1,43)=4.51, P=.039$, respectively], emerged. Inspection of the means presented in Table 2 suggests that, in general, 
TABLE 2

Means and (Standard Deviations) for Task Difficulty Ratings in Function of Valence and Intensity of the Decoded Emotion Display as well as Presentation Condition

\begin{tabular}{lccccc}
\hline & \multicolumn{2}{c}{ Mixed Presentation } & & \multicolumn{2}{c}{ Block Presentation } \\
\cline { 2 - 3 } Intensity & Angry & Happy & & Angry & Happy \\
\hline Strong & $44.86(34.09)$ & $17.50(14.80)$ & & $40.18(37.21)$ & $21.66(21.87)$ \\
Weak & $58.64(42.50)$ & $54.25(39.97)$ & & $56.20(49.70)$ & $37.53(33.50)$ \\
\hline
\end{tabular}

weak expressions were perceived as more difficult to decode than strong expressions. Also, happy faces were perceived as easer to decode than angry faces. However, for weak expressions this was the case only in the block presentation condition, whereas in the mixed condition the weak expressions of happiness were perceived as equal in difficulty to the weak expressions of anger.

\section{Facial EMG}

Corrugator Supercilii. Activity of the Corrugator Supercilii was measured to assess the drawing together of the eyebrows in frowning. Presence of relatively more Corrugator Supercilii activity for anger than for happy expressions would be indicative of congruent facial expressions.

Significant main effects of Emotion $[F(1,40)=22.68 ; P<.001]$, and Intensity $[F(1,40)=8.98 ; P=.005]$, as well as a marginally significant Emotion $\times$ Intensity interaction $[F(1,40)=3.04 ; P=.089]$ emerged. Inspection of the means shows more Corrugator Supercilii activity while decoding angry expressions than while decoding happy expressions (see Fig. 1). Further, participants showed more Corrugator Supercilii activity when assessing weak then when assessing intense expressions; however, this difference is only significant for the judgement of happy expressions. No effect for Presentation emerged.

To assess the influence of task difficulty on Corrugator Supercilii activity, a 2 (Emotion: angry vs. happy; within-subjects) $\times 2$ (Intensity of expression: strong vs. weak; within-subjects) $\times 2$ (mixed vs. block Presentation; between-subjects) mixed model analysis of covariance with self-rated task difficulty as covariate was conducted using a multivariate approach. None of the regression coefficients reached significance. However, eta $^{2}$ for the Intensity factor dropped from $18 \%$ to $8 \%$, suggesting that the effect of the intensity manipulation on Corrugator Supercilii is partly due to differences in the difficulty of the task. 
Mixed Presentation

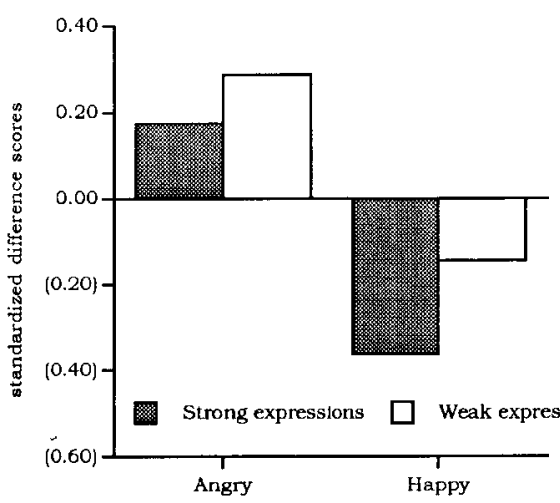

Emotion
Block Presentation

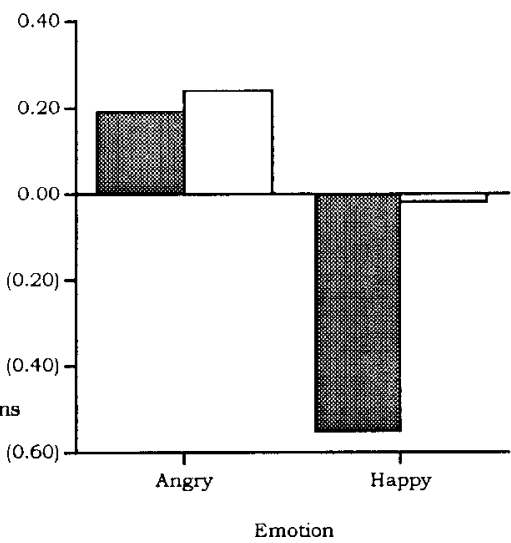

FIG. 1. Mean Corrugator Supercilii EMG in function of the valence and intensity of the decoded emotion display as well as presentation condition (standardised difference scores).

Zygomaticus Major. No significant main effects or interactions emerged.

\section{Discussion}

In summary, congruent facial expressions were found for anger expressions for both types of presentation: when judging expressions of anger participants showed more Corrugator Supercilii activity than when judging expressions of happiness. Further, participants show more Corrugator Supercilii activity when judging weak than when judging strong expressions of both happiness or anger. Also, participants showed less Corrugator Supercilii activity when judging strong happy expressions than when judging weak happy expressions. No effects for Zygomaticus Major were found. Facial muscle activity was not influenced by the order of presentation of the stimuli. The difficulty ratings support the notion that expressions of anger were more difficult to decode than were expressions of happiness and that, in general, weak expressions were more difficult to decode than strong expressions. The results from the analysis of covariance including task difficulty as covariate are in concordance with the notion that the effects of the intensity of the expressions on Corrugator Supercilii activity was partly due to an effect of task difficulty.

Because no effect of Presentation was found, the data do not support the Mood Induction Hypothesis. However, the pattern of results is congruent 
with both remaining hypotheses. More Corrugator Supercilii activity was found for weak than for strong expressions. This finding favours the Difficulty Hypothesis. However, the Mimicry Hypothesis is supported by the fact that more Corrugator Supercilii activity was found while observers were judging angry expressions than when judging happy expressions. Yet, this pattern of results could also be accounted for by the Difficulty Hypothesis, as angry expressions are more difficult to decode than happy expression. The absence of effects on the Zygomaticus Major site apparently argues against the Mimicry Hypothesis, suggesting that the pattern of results can be completely accounted for by the Difficulty Hypothesis. However, the failure to find differences in Zygomaticus Major activity as a function of the emotion decoded is somew hat inconclusive. EMG activity recorded on the Zygomaticus Major site may result not only from Zygomaticus Major activity per se, but also from Masseter activity (e.g. Hess et al., 1992). Hence, anger-congruent Masseter activity during exposure to angry faces could have been recorded at the Zygomaticus Major site at levels similar to Zygomaticus major activity during exposure to happy faces.

In conclusion, the pattern of results of Experiment 1 argues against the Mood Induction Hypotheses, but it does not disentangle the Mimicry and the Difficulty Hypotheses.

\section{EXPERIMENT 2}

The previous discussion notes that testing the relative merits of the Mimicry versus the Difficulty Hypothesis requires an experimental manipulation that permits the variation of task difficulty independently of the effects of the emotional quality of the stimuli (anger vs. happiness). The Difficulty Hypothesis predicts that participants frown at difficult-to-decode expressions of both anger and happiness, whereas they smile or relax in reaction to easy-to-decode expressions of both emotions. Conversely, the Mimicry Hypothesis predicts that participants frown at anger displays and smile at happy displays, independent of the task difficulty manipulation.

Experiment 2 was designed to manipulate decoding difficulty and emotional nature of the stimuli independently. This manipulation was based on two premises: (1) the distinction between expressions of positive and negative affect is generally easy to make; and (2) the distinction between posed and spontaneous expressions is generally difficult to make. Thus, we varied task difficulty by asking the participants to distinguish between posed and spontaneous expressions for expressions of either negative or positive affect. Specifically, in one condition (the Complex Positive Condition) ratings of happy expressions were made difficult, and ratings or angry expressions were made easy, but asking participants to judge 
whether the expression displayed was indicative of: (a) negative affect, (b) spontaneous positive affect, or (c) posed positive affect. In the other condition (the Complex Negative Condition) rating difficulty was reversed by asking participants to judge whether the expression displayed was indicative of: (a) positive affect, (b) spontaneous negative affect, or (c) posed negative affect.

In addition, to overcome the difficulty in interpreting activity measured at the Zygomaticus Major site in Experiment 1, analysis of Zygomaticus Major activity was replaced by analysis of activity at the Orbicularis Oculi site, which is also involved in happy expressions (see e.g. Duchenne, 1990/1862; Ekman \& O'Sullivan, 1991; Frank, Ekman, \& Friesen, $1993)$.

Experiment 2 actually consists of two studies, designated hereafter as Experiment $2 \mathrm{a}$ and Experiment 2b, which were conducted in two laboratories. The procedures employed will therefore be described separately. Experiment $2 \mathrm{a}$ comprised the emotion and task difficulty manipulation $\mathrm{s}$ for the block presentation condition. Experiment $2 b$ comprised the same manipulations for the mixed-order presentation condition. In addition, to assess comparability between laboratories, data for the Complex Positive Judgement task in the mixed-order condition were collected as part of both Experiments $2 \mathrm{a}$ and $2 \mathrm{~b}$. As these two experiments represent different cells of a more complex design, they will be analysed together.

\section{Method}

\section{Experiment 2a}

\section{Overview}

Experiment $2 \mathrm{a}$ comprised the two task difficulty manipulations for the block presentation condition. In addition, to assess comparability with the data collected in Experiment $2 \mathrm{~b}$ in another laboratory, data were also collected for the Complex Positive Judgement task in the mixed presentation condition.

\section{Participants}

Sixty female volunteers were recruited at the University of Quebec at Montreal. Forty individuals participated in the block presentation condition, half of whom performed the Complex Positive Judgement task and the other half performed the Complex Negative Judgement task. Futher, 20 participants completed the Complex Positive Judgement task in the mixed presentation condition. 


\section{Dependent Measures}

Facial EMG. Activity of the Zygomaticus Major, Corrugator Supercilii, as well as Orbicularis Oculi (to assess the eye wrinkles typical of a veridical smile) and Levator Labii Alesque Nasii (to assess the curling of the upper lip) was measured. Electrode placements were chosen according to Fridlund and Cacioppo (1986). The same procedure and equipment as in Experiment 1 were employed.

Ratings. Participants were asked to indicate to which of three categories the emotion portrayed corresponded; specifically, participants had to discriminate either between negative affect, felt happiness and posed happiness (Complex Positive Judgement condition) or between positive affect, felt negative affect, and posed negative affect (Complex Negative Judgement condition).

Furthermore, to assess task difficulty, participants were asked to indicate on a scale (resolution: 0-200) how difficult the task was.

Latency Times. The time participants needed to complete the decoding task was measured. One should note that these latency times are not reaction times. Participants were not asked to answer as quickly as possible in order to not interfere with mimicry. Thus, the latency times correspond to the time from the appearance of the emotional facial expression on the screen to the moment the participants finished the rating task. ${ }^{3}$

\section{Procedure}

The same general procedure as in Experiment 1 was employed, with the same stimulus set. However, because we measured EMG at more muscle sites (four sites instead of two) we adapted the cover story: The experimenter explained to the participants that the experiment was concerned with their facial temperature.

\section{Experiment $2 b$}

\section{Overview}

In Experiment $2 b$ the data collected were for the mixed presentation condition using the same procedure, stimuli, and dependent measures as Experiment 2a.

Latency time correlated highly with self-reported task difficulty and is thus not reported in this context. 


\section{Participants}

Forty female participants, 20 for the Complex Positive Judgement condition and 20 for the Complex Negative Judgement condition, were recruited at the University of Louvain at Louvain-la-Neuve, Belgium. Unfortunately, due to a hard-disk failure, physiological data of only 30 participants (16 in the Complex Negative Judgement condition and 14 in the Complex Positive Judgement condition) could be used for the analyses. All participants received course credits for their participation.

\section{Dependent Measures}

Facial EMG. Facial EMG was measured on the left side of the face. Activity of the Zygomaticus Major was employed to assess smiling, activity of the Corrugator Supercilii was employed to assess frowning, and activity of the Orbicularis Oculi indexed the eye wrinkles typical of veridical smiles. Muscular activity was measured using bipolar placements of Med Associates Inc. Ag/ $\mathrm{AgCl}$ miniature surface electrodes with Med Associates Inc. electrolyte gel (TD41). The skin was cleansed with isopropyl alchohol and lightly abraded with an ink-eraser. Electrode placements were chosen according to Fridlund and Cacioppo (1986). A custombuilt coupler with a $50 \mathrm{~Hz}$ notch filter was used to amplify the raw EMG signals, which were integrated with $100 \mathrm{msec}$ time constant. The smoothed EMG signal was sampled at $10 \mathrm{HZ}$ and stored to disk.

\section{Results ${ }^{4}$}

\section{Analyses}

The dependent variables were entered into a 2 (Emotion: angry vs. happy; within-subjects) $\times 2$ (mixed vs. block presentation; between-subjects) $\times 2$ (Task difficulty: complex positive judgement vs. complex negative judgement; between-subjects) mixed model analysis of variance using a multivariate approach.

\section{Manipulation Check}

To assess the effectiveness of the task difficulty manipulation, the selfreported task difficulty ratings were analysed. An Emotion $\times$ Task difficulty interaction was expected, because in the Complex Positive Judgement

${ }^{4}$ For the purpose of facilitating the presentation of the following analyses the data from the weak expressions were not included. The pattern of results does not change notably when the data is included. 
condition angry faces were supposed to be easier to decode than happy faces, while the reverse should be the case for the Complex Negative Judgement condition. As expected, a significant Emotion $\times$ Task difficulty interaction emerged $[F(1,84)=16.71, P<.001]$. However, main effects of Task difficulty and Emotion also emerged $[F(1,84)=15.14, P<.001$ and $F(1,84)=6.43, P=.013]$, respectively. Overall, Complex Negative Judgements were perceived as more difficult than Complex Positive Judgements; furthermore, angry faces were generally perceived as more difficult to decode than happy faces. In addition, the Emotion $\times$ Task difficulty interaction was qualified by an Emotion $\times$ Task difficulty $\times$ Presentation-type interaction $[F(1,84)=7.61, P=.007]$ (see Table 3 ). Post-hoc analyses revealed that in the mixed presentation condition, Complex Negative Judgements were always more difficult than Complex Positive Judgements, regardless of the emotional nature of the stimulus $[F(1,47)=9.32, P=.004]$. In the block presentation condition, the data conformed to the expected Task difficulty $\times$ Emotion interaction $[F(1,38)$ $=34.04, P<.001]$, although judgements in the Complex Negative Condition and Judgements of angry faces tended to be perceived as more difficult $[F(1,38)=6.12, P=.018 ; F(1,38)=11.32, P=.002$, respectively $] .^{5}$

In sum, in the block presentation condition the difficulty manipulation had the predicted effect. In the mixed presentation condition, Complex Negative Judgements were always perceived as more difficult. However, because there are no differences in perceived task difficulty for the judgements of angry and happy faces within difficulty conditions, EMG data can be compared within each difficulty condition without confounding Emotion and Task difficulty effects. This difference in patterns of difficulty ratings between presentation conditions suggests that different judgement processes might have been active in these conditions. This point will be further developed in the Discussion.

\section{Comparison Across Laboratories}

To assess the comparability of the physiological data collected by the two laboratories, a profile analysis was conducted on the EMG data of the mixed presentation, Complex Positive Judgement condition which was run in both laboratories. For this, the pattern of results for the two muscles sites and the two emotion conditions was compared across laboratories. Parallel profiles indicate that the same differences between each successive two measures were found in both laboratories (for more detail regarding profile analyses see Stevens, 1992). The results from this analysis are compatible with the notion that the profiles are parallel $[F(3,29)=.48, P>.70]$.

\footnotetext{
The analysis of the latency times corroborated this pattern of results.
} 
TABLE 3

Means and (Standard Deviations) for Task Difficulty Ratings as a Function of Presentation Type, Emotion, and Manipulated Task Difficulty

\begin{tabular}{lccccc}
\hline & \multicolumn{2}{c}{ Mixed Presentation } & & \multicolumn{2}{c}{ Block Presentation } \\
\cline { 2 - 3 } Difficulty & Angry & Happy & & Angry & Happy \\
\hline Positive Complex & $30.08(34.07)$ & $31.03(27.63)$ & & $21.09(15.47)$ & $34.56(23.42)$ \\
Negative Complex & $58.08(44.69)$ & $46.69(39.95)$ & & $69.53(44.04)$ & $19.43(16.48)$ \\
\hline
\end{tabular}

\section{Facial EMG}

Corrugator Supercilii. The standardised EMG difference scores were entered into a 2 (Emotion: within-subjects) $\times 2$ (Presentation type; between-subjects) $\times 2$ (Task difficulty; between-subjects) mixed model analysis of varience using a multivariate approach. A main effect of emotion emerged: exposure to angry faces induced more Corrugator Supercilii activity than exposure to happy faces $[F(1,84)=11.18, P<$ $.001]$. This main effect is qualified by a Task difficulty $\times$ Emotion interaction $[F(1,84)=4.66, P=.034]$, which is qualified by a Task difficulty $\times$ Emotion $\times$ Presentation type interaction $[F(1,84)=4.81$, $P=.031]$. The pattern of these interactions is illustrated in Fig. 2. In the block condition, Corrugator Supercilii activity varies as a function of task difficulty: Participants show more Corrugator Supercilii activity in response to angry faces in the Complex Negative Condition than in the Complex Positive Condition, whereas the reverse was found for happy faces.

This pattern is confirmed by the results of a 2 (Emotion; withinsubjects) $\times 2$ (Task difficulty; between-subjects) analysis of variance, using a multivariate approach, conducted on the data from the block condition only. This analysis revealed a Emotion $\times$ Task difficulty interaction $[F(1,84)=10.36, P=.003]$, which explains $22 \%$ of the variance. Furthermore, when self-rated task difficulty is introduced as a covariate, the Emotion $\times$ Task difficulty interaction becomes nonsignificant $[F(1,36)=3.06, P=.089]$. Also, $37 \%$ of the variance in Corrugator Supercilii activity in the block condition is explained by self-rated task difficulty. This pattern of results is congruent with the Difficulty Hypothesis

A different pattern of results emerged for the mixed presentation condition. A 2 (Emotion; within-subjects) $\times 2$ (Task difficulty; betweensubjects) analysis of variance using a multivariate approach revealed a significant main effect of Emotion $[F(, 84)=9.64, P=.003]$. Exposure 
Mixed Presentation

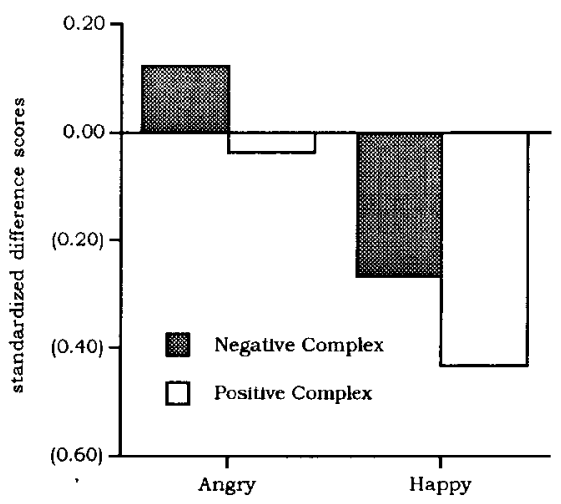

Emotion
Block presentation

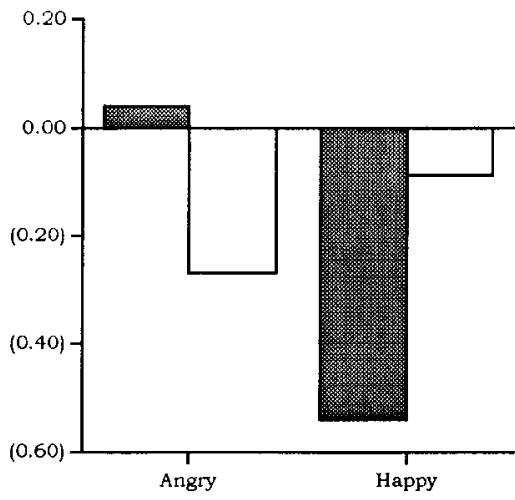

Emotion

FIG. 2. Mean Corrugator Supercilii EMG in function of the valence of the decoded emotion display, manipulated task difficulty, and presentation condition (standardised difference scores).

to angry faces induced more Corrugator Supercilii activity than did exposure to happy faces. As the manipulation check suggested no confound between task difficulty and emotion in the mixed condition, this effect can be interpreted as a pure effect of emotion, which is congruent with the Mimicry Hypothesis. Moreover, when self-rated task difficulty is employed as a covariate, the pattern of results does not change and the emotion main effect remains significant $[F(1,46)=10.93, P=.002]$. Also, for this condition only $1.5 \%$ of the variance in Corrugator Supercilii activity can be explained by perceived task difficulty.

Orbicularis Oculi. The standardised difference scores were entered into a 2 (Emotion) $\times 2$ (Presentation type) $\times 2$ (Task difficulty) analysis of variance using a multivariate approach. A significant Emotion $\times$ Presentation type interaction $[F(1,84)=8.49 ; P=.005]$, and a significant Task difficulty $\times$ Presentation type interaction $[F(1,84)=4.64 ; P=.033]$ emerged (see Fig. 3). A 2 (Emotion) $\times 2$ (Task difficulty) analysis of variance conducted separately for the block condition shows no significant main effects or interactions for Orbicularis Oculi, a finding which is congruent with the Difficulty Hypothesis. For the mixed condition, on the other hand, the post-hoc analysis revealed a significant main effect of Emotion $[F(1,84)=10.88, P=.002]$; participants showed more Orbicularis Oculi activity when exposed to happy than when exposed to angry faces. This finding is congruent with the Mimicry Hypothesis. 
Mixed Presentation

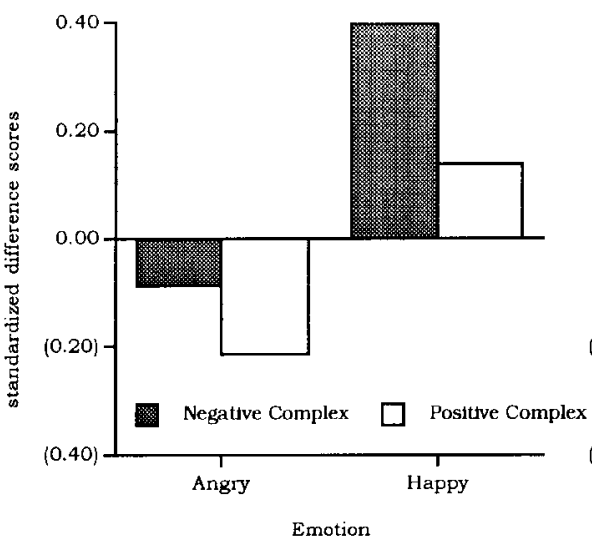

Block Presentation

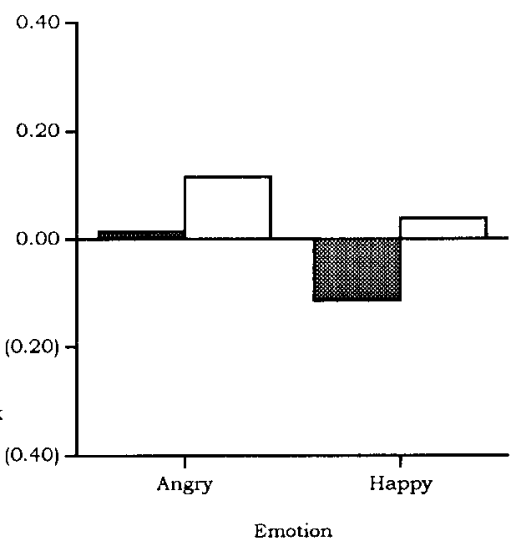

FIG. 3. Mean Orbicularis Oculi EMG in function of valence of the decoded emotion display, manipulated task difficulty, and presentation condition (standardised difference scores).

\section{GENERAL DISCUSSION}

The goal of this study was to assess the process underlying facial reactions to the emotion displays of others. Three hypotheses were proposed. (1) According to the Mood Induction Hypothesis, observers' congruent facial expressions reflect a mood induced by the repeated exposure to a specific type of facial expression. (2) According to the Mimicry Hypothesis, observers' congruent facial expressions represent facial mimicry as part of the empathic process underlying the decoding of emotional facial expressions. (3) According to the Difficulty Hypothesis congruent facial expressions are on artefact of the fact that angry expressions are more difficult to decode than happy expressions. This hypothesis interprets frowns as markers of cognitive load. Thus, the first two hypotheses view congruent facial expressions as a marker of an affective reaction to the emotional displays of others, whereas the third hypothesis views them as a marker of cognitive load. A series of experiments was conducted to investigate the three hypotheses. Experiment 1 allowed the rejection of the Mood Induction Hypothesis but was inconclusive with regard to the other two hypotheses. The findings from Experiment 2 suggest support for both remaining hypotheses. Specifically, the findings from the mixed presentation condition support the Mimicry Hypothesis whereas the findings from the block presentation condition favour the Difficulty Hypothesis. 
How can we explain these different findings for the two conditions? A closer look at the decoding task shows that participants in the mixed condition were faced with two types of judgements. First, they had to decide whether a given expression was indicative of a positive or a negative affect. Second, they had to decide for one type of expression (depending on the task difficulty manipulation), whether the expression was spontaneous or posed. Some evidence suggests that this last decision may be made using a patter-matching procedure whereby the participant searches for the presence of specific cues for posing and that these cues may be the same for different emotions (Hess \& Kleck, 1994). Participants in the block condition, on the other hand, saw a series of the same type of expression for each of three emotions. Thus, these participants needed to perform an affective judgement regarding the valence of the target persons' expressions only once-for the first of these expressions. For the other expressions in the series, the participants' task was reduced to the assessment of the genuineness of the expression only. This is different from the participants' task in Experiment 1, where no difference between condition s emerged. In Experiment 1, participants had to assess each expression using an emotion profile with graded intensity ratings. Thus, participants in the block condition, although aware of the predominant emotion expressed, where required to assess the possible presence of other emotions for every expression in the series.

One may therefore speculate that all participants in Experiment 1 and the participants in the mixed condition in Experiment 2 were required to make both affective and nonaffective judgements of the targets' emotional expressions, whereas participants in the block condition of Experiment 2 had to make mainly nonaffective judgements. Thus, the present results suggest that facial mimicry occurs only when affective judgements are required. When other types of judgements are performed, facial responses are not reflective of mimicry but rather of the cognitive load imposed by the judgement task. In summary, the present findings suggest that facial expressions in response to others' facial emotional displays may reflect both affective and cognitive processes.

The present findings have implications for our conceptualisation of processes linked to the recognition of others' emotional states, such as empathy and counter-transference. Because facial mimicry may be conceived of as a marker of an empathic emotion recognition process (Hoffman, 1984; Lipps, 1907), the present study suggests that empathy may occur only during certain types of judgements regarding others' emotional expressions. In particular, empathy may not occur when the observer is not attending to the emotional quality of an interaction partner's expression (e.g. when questioning its genuineness). This notion is congruent with concerns expressed in the context of humanistic psychotherapy regarding the 
therapist's ability to show reflective empathy (Rogers, 1951). According to this approach, therapists should abstain from questioning the genuineness of the client's expressed feelings in order not to endanger the establishment of rapport through reflective empathy.

Manuscript received 6 May 1996

Revised manuscript received 13 June 1997

\section{REFERENCES}

Banse, R. (1995). Physio3 (PC software). Humboldt University: Berlin.

Basch, M.F. (1983). Empathic understanding: a review of the concept and some theoretical considerations. Journal of the American Psychoanalytical Association, 31, 101-126.

Bavelas, J.B., Black, A., Lemery, C.R., \& Mullett, J. (1986). "I show how you feel": Motor mimicry as a communicative act. Journal of Personality and Social Psychology, $50,322-329$.

Bavelas, J.B., Black, A., Lemery, C.R., \& Mullett, J. (1987). Motor mimicry as primitive empathy. In N. Eisenberg \& J. Strayer (Eds.), Empathy and its development (pp. $317-$ 338). New York: Cambridge University Press.

Bem, D.J. (1972). Self-perception theory. In L. Berkowitz (Ed.), Advances in experimental social psychology (Vol. 6, pp. 1-62). New York: Academic Press.

Berger, S.M., \& Hadley, S.W. (1975). Some effects of a model's performance on an observers' electromyographic activity. Journal of Psychology, 88, 262-276.

Bernieri, F.J., \& Rosenthal, R. (1991). Interpersonal coordination: Behavior matching and interactional synchrony. In R.S. Feldman \& B. Rimé (Eds.), Fundamentals of nonverbal behavior (pp. 401-432). New York: Cambridge University Press.

Bourgeois, P. Couture, J., Herrera, P., Blairy, S., Hess, U., \& Philippot, P. (1995, October). Induction des émotions par rétroaction faciale et respiratoire [Induction of emotion by facial and respiratory feedback]. Present at the 18th annual meeting of the Societe Québécoise pour la Recherche en Psychologie, Ottawa, Cananda.

Bush, L.K., Barr, C.L., McHugo, G.J., \& Lanzetta, J.T. (1989). The effects of facial control and facial mimicry on subjective reactions to comedy routines. Motivation and Emotion, $13,31-52$.

Cacioppo, J.T., Petty, R.E., Losch, M.E., \& Kim, H.S. (1986). Electromyographic activity over facial muscle regions can discriminate the valence and intensity of affective reactions. Journal of Personality and Social Psychology, 50, 260-268.

Darwin, C. (1965). The expression of the emotions in man and animals. Chicago, IL: University of Chicago Press. (Original work published 1872)

Dimberg, U. (1982). Facial reactions to facial expressions. Psychophysiology, 19, 643-647.

Dimberg, U. (1987). Facial reactions, autonomic activity and experienced emotion: A three component model of emotional conditioning. Biological Psychology, 24, 105-122.

Duchenne, G.B. (1990). The mechanism of human facial expression (R.A. Cuthbertson, Ed. and Trans.). Cambridge: Cambridge University Press. (Original work published, 1862)

Duclos, S.E., Laird, J.D., Schneider, E., Sexter, M., Stern, L., \& Van Lighten, O. (1989). Categorial vs. dimensional effects of facial expressions on emotional experience. Journal of Personality and Social Psychology, 57, 100-108.

Eisenberg, N., \& Strayer, J. (1987). Critical issues in the study of empathy. In N. Eisenberg \& J. Strayer (Eds.), Empathy and its development (pp. 3-13). New York: Cambridge University Press. 
Eisenberg, N., \& Fabes, R.A. (1990). Empathy, conceptualization, measurement, and relation to prosocial behavior. Motivation and Emotion, 14, 131-149.

Ekman, P., Friesen, W.V., \& Ellsworth, P (1972). Does the face provide accurate information? In P. Ekman (Ed.), Emotion in the human face (pp. 56-97). New York: Cambridge University Press.

Ekman, P., \& O’Sullivan, M. (1991). Facial expression: Methods, means, and moues. In R.S. Feldman \& B. Rimé (Eds.), Fundamentals of nonverbal behavior (pp. 163-199). New York: Cambridge University Press.

Feldman, R.S., \& Rimé, B. (1991). (Eds.), Fundamentals of nonverbal behavior. New York: Cambridge University Press.

Frank, M.G., Ekman, P., \& Friesen, W.V. (1993). Behavioral markers and recognizability of the smile of enjoyment. Journal of Personality and Social Psychology, 64, 83-93.

Fridlund, A.J., \&. Cacioppo., J.T. (1986). Guidelines for human electromyographic research. Psychophysiology, 23, 567-589.

Fridlund. A.J., Ekman, P., \& Oster, H. (1987). Facial expressions of emotion: Review of the literature, 1970-1983. In A.W. Siegman \& S. Feldstein (Eds.), Nonverbal behavior and communication (pp. 143-224). Hillsdale, NJ: Lawrence Erlbaum Associates Inc.

Hatfield, E., Cacioppo, J.T., \& Rapson, R.L. (1992). Emotional contagion In M.S. Clark (Ed.), Review of personality and social psychology: Vol. 14. Emotion and social behavior (pp. 151-177). Newbury Park, CA: Sage.

Hatfield, E., Hsee, C.K., Costello, J., Weisman, M.S., \& Denney, C. (1995). The impact of vocal feedback on emotional experience and expression. Journal of Social Behavior and Personality, 10, 293-312.

Hess, U., Kappas, A., \& Banse, R. (1995). The intensity of facial expressions is determined by underlying affective state and social situation. Journal of Personality and Social Psychology, 69, 280-288.

Hess, U., Kappas, A., McHugo, G.J., Lanzetta, J.T., \& Kleck, R.E. (1992). The facilitative effect of facial expression on the self-generation of emotion. International Journal of Psychophysiology, 12, 251-265.

Hess, U., Kappas, A., \& Scherer, K.R. (1988). Multichannel communication of emotion: Synthetic signal production. In K.R. Scherer (Ed.), Facets of emotion: Recent research. Hillsdale, NJ: Lawrence Erlbaum Associates Inc.

Hess, U., \& Kleck, R.E. (1994). The cues decoders use in attempting to differentiate emotion elicited and posed facial expressions. European Journal of Social Psychology, 24, 367-381.

Hoffman, M.L. (1984). Interaction of affect and cognition on empathy. In C.E. Izard, J. Kagan, \& R.B. Zajonc (Eds.), Emotions, cognition, and behavior (pp. 101-131). New York: Cambridge University Press.

Hsee, C.K., Hatfield, E., \& Chemtob, C. (1992). Assessments of the emotional states of others: Conscious judgments versus emotional contagion. Journal of Social and Clinical Psychology, 11, 119-128.

James, E. (1890). Principles of psychology. New York: Holt.

Kappas, A., Hess, U., \& Banse, R. (1992). Skin conductance reactions to dynamic facial expressions revisited: Empathic responding or information processing? Psychophysiology, 29, S42 (Abstract)

Krause, R. (1990). Über die echten und unechten Emotionen und andere Merkwürdigkeiten. Paper presented at the 37 th Conference of the Deutsche Gesellschaft für Psychologie, Kiel, Germany.

Krause, R., \& Lütolf, P. (1988). Facial indicators of transference processes within psychoanalytic treatment. In H. Dahl \& H. Kächele (Eds.), Psychoanalytic process research strategies (pp. 258-272). Heidelberg: Springer. 
Laird, J.D., Alibozak, T., Davainis, D., Deignan, K., Fontanella, K., Hong, J., Levy, B., \& Pacheco, C. (1994). Individual differences in the effects of spontaneous mimicry on emotional contagion. Motivation and Emotion, 18, 231-247.

Levenson, R.E., \& Ruef, A.M. (1992). Empathy: A physiological substrate. Journal of Personality and Social Psychology, 63, 234-246.

Lipps, T. (1907). Das Wissen von fremden Ichen. In T. Lipps, Psychologische Untersuchungen (Vol. 1). Leipzig: Engelmann.

Manstead, A.S.R. (1988). The role of facial movement in emotion. In H.L. Wagner (Ed.), Social psychophysiology: Theory and clinical application (pp. 105-129). Chichester, UK: Wiley.

Matsumoto, D. (1987). The role of facial response in the experience of emotion: More methodological problems and a meta-analysis. Journal of Personality and Social Psychology, 52, 759-768.

Matsumoto, D., \& Ekman, P. (1988). Japanese and Caucasian facial expressions of emotion (JACFEE) and neutral faces (JACNeuf). San Francisco: San Francisco State University.

Meltzoff, A.N. (1990). Foundations for developing a concept of self: The role of imitation in relating self to other and the value of social mirroring, social modeling and self practice in infancy. In D. Cicetti \& M. Beeghly (Eds.), The self in transition: Infancy to childhood (pp. 139-164). Chicago, IL: University of Chicago Press.

Noller, P. (1985). Video primacy-A further look. Journal of Nonverbal Behavior, 9, 28-47.

Patterson, M.L. (1991). A functional approach to nonverbal exchange. In R.S. Feldman \& Rimé (Eds.), Fundamentals of nonverbal behavior. New York: Cambridge University Press.

Philippot, P., Chapelle, G., \& Blairy, S. (1994, July). Respiratory feedback and the generation of emotion. Paper presented at the VIIIth Meeting of the International Society for Research on Emotion, Cambridge, UK.

Rogers, C.R. (1951). Client centered therapy: Its current practice, implications, and theory. Boston, MA: Houghton-Mifflin.

Schneider, F., Gur, R.C., Gur, R.E., \& Muenz, L. (1994). Standardized mood induction with happy and sad facial expressions. Psychiatry Research, 51, 19-31.

Smith, C.A. (1989). Dimensions of appraisal and physiological response in emotion. Journal of Personality and Social Psychology, 56, 339-353.

Stepper, S., \& Strack, F. (1993). Proprioceptive determinants of affective and nonaffective feelings. Journal of Personality and Social Psychology, 64, 211-220.

Stevens, J. (1992). Applied multivariate statistics for the social sciences (2nd ed.). Hillsdale, NJ: Lawrence Erlbaum Associated Inc.

Strayer, J. (1993). Children's concordant emotions and cognitions in response to observed emotions. Child Development, 64, 188-201.

Velten, E. (1968). A laboratory task for the induction of mood states. Behavior Research and Therapy, 6, 473-482.

Wallbott, H.G. (1991). Recognition of emotion from facial expression via imitation? Some indirect evidence for an old theory. British Journal of Social Psychology, 30, 207-219.

Winton, W.M. (1986). The role of facial response in self-reports of emotion: A critique of Laird. Journal of Personality and Social Psychology, 50, 808-812. 